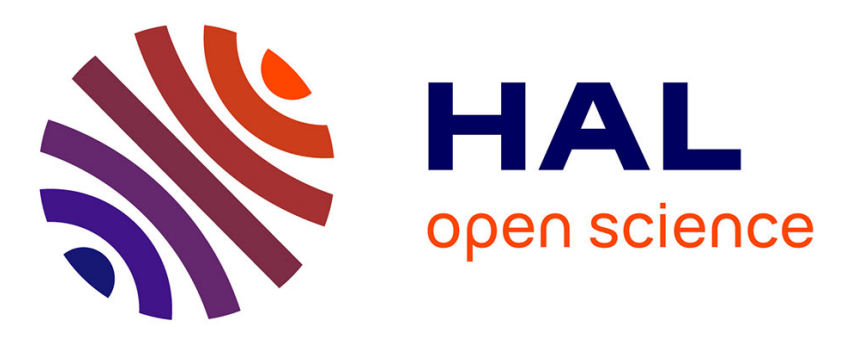

\title{
Signatures of farther neighbor couplings in artificial square ice
}

O. Brunn, Y. Perrin, Benjamin Canals, N. Rougemaille

\section{To cite this version:}

O. Brunn, Y. Perrin, Benjamin Canals, N. Rougemaille. Signatures of farther neighbor couplings in artificial square ice. Physical Review B, 2021, 103 (9), pp.094405. 10.1103/physrevb.103.094405. hal-03386485

\section{HAL Id: hal-03386485 \\ https://hal.science/hal-03386485}

Submitted on 19 Oct 2021

HAL is a multi-disciplinary open access archive for the deposit and dissemination of scientific research documents, whether they are published or not. The documents may come from teaching and research institutions in France or abroad, or from public or private research centers.
L'archive ouverte pluridisciplinaire HAL, est destinée au dépôt et à la diffusion de documents scientifiques de niveau recherche, publiés ou non, émanant des établissements d'enseignement et de recherche français ou étrangers, des laboratoires publics ou privés. 


\title{
Signatures of farther neighbor couplings in artificial square ice
}

\author{
O. Brunn $\odot,{ }^{1,2,3}$ Y. Perrin $\odot,{ }^{1}$ B. Canals, ${ }^{1}$ and N. Rougemaille $\odot^{1}$ \\ ${ }^{1}$ Université Grenoble Alpes, CNRS, Grenoble INP, Institut NEEL, 38000 Grenoble, France \\ ${ }^{2}$ Institute of Scientific Instruments of the Czech Academy of Sciences, Královopolská 147, 61264 Brno, Czech Republic \\ ${ }^{3}$ Institute of Physical Engineering, Brno University of Technology, Technická 2, 61669 Brno, Czech Republic
}

(Received 12 October 2020; revised 2 February 2021; accepted 16 February 2021; published 2 March 2021)

\begin{abstract}
The analysis of the magnetic structure factor obtained after imaging a field-demagnetized artificial square ice reveals qualitative deviations from what would predict the six-vertex model. More specifically, additional features appear for certain $q$ vectors in the experimental magnetic structure factor that are absent in the theoretical one. Using Monte Carlo simulations, we demonstrate that these features originate from dipolarlike, farther neighbor couplings. Our results thus show that the long-range magnetostatic interactions coupling the nanomagnets are not totally washed out in a field-demagnetized artificial square ice and cannot be neglected as they impact the magnetic correlations within or at the vicinity of the ice manifold. Artificial square ice systems are then expected to ultimately order, provided that the ground state can be approached.
\end{abstract}

DOI: 10.1103/PhysRevB.103.094405

\section{INTRODUCTION}

Two-dimensional arrays of interacting magnetic nanostructures are now well-established model systems to explore the physics of highly frustrated magnets [1-3]. Complementing what can be done with chemically synthesized compounds [4,5], artificially made spin lattices offer a laboratory-on-chip approach: almost any kind of geometry can be designed [3,6-9], magnetic interactions can be tuned [10-13], structural defects can be engineered [14], thermal fluctuations are adjustable in the desired temperature range [15-17], the spin degree of freedom can be controlled [18-20], etc. Combined with the capability to image spin configurations directly in real space, at the scale of a nanomagnet, artificial spin systems can be viewed as experimental simulators of frustrated magnetism.

Among the works done so far, many studies on artificial spin systems have been devoted to the square geometry $[10,15,21-26]$. This geometry was first proposed to realize the so-called square ice model [27], a model capturing the low-energy physics of water ice, but in two dimensions [1,2]. However, two-dimensional square arrays of interacting nanomagnets do not show the expected disordered behavior: they order in an antiferromagnetic fashion because of the inequivalent coupling strengths between collinear and perpendicular nanomagnets [21].

Several modifications of the square lattice have been proposed, theoretically [28-31] and experimentally [6,10,25,26], to recover the ice degeneracy of the ground state. This could be done, for example, by shifting vertically one of the two sublattices of the square lattice. Doing so, the coupling strength $J_{2}$ [shown in green in Fig. 1(a)] between collinear nanomagnets remains unchanged, whatever the amplitude of the vertical shift $h$. However, the coupling strength $J_{1}$ [shown in red in Fig. 1(a)] between orthogonal nanomagnets is varied continuously [see Fig. 1(b)] and can be made even negligible for large $h$ values. The vertical shift $h$ is thus an experimental knob one can play with to adjust $J_{1}$, especially to reach the $J_{1}=J_{2}$ condition required in the square ice model. This was done for both athermal and thermally active arrays of nanomagnets, and the extensive degeneracy of the ice manifold was recovered $[10,26]$.

\section{MOTIVATION}

Generally, artificial spin systems consist in the arrangement of nanomagnets coupled through magnetostatics. Thus they are dipolar systems by design, and considering nearestneighbor interactions only to describe their physics is an approximation. In some systems, this approximation fails to capture the physics observed experimentally. This is the case for the artificial kagome ice [14,16,32-38] and the artificial kagome Ising antiferromagnet [39-41], which develop a lowenergy physics that cannot be accounted for by considering solely nearest-neighbor interactions $[42,43]$.

Artificial square ice systems, however, seem to be well approximated by a nearest-neighbor spin Hamiltonian [10,26]. We can then wonder whether or not the presence of longrange dipolar interactions can be evidenced experimentally in an artificial square ice, or if they are totally washed out in practice, for example, because of intrinsic disorder in the fabricated arrays or because they are made invisible due to an inefficient energy minimization protocol. This is an important question, as dipolar interactions are known to lift the extensive degeneracy of the square ice manifold and to ultimately order the system $[28,29]$.

To address this issue, we fabricated a square ice system using a technique we developed previously [10]. The lattices were field demagnetized multiple times and the resulting magnetic configurations imaged using magnetic force 
(a)

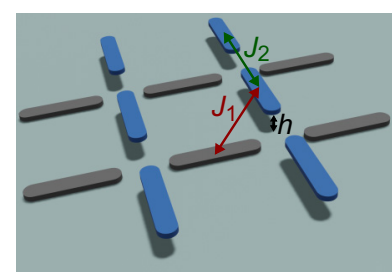

(b)

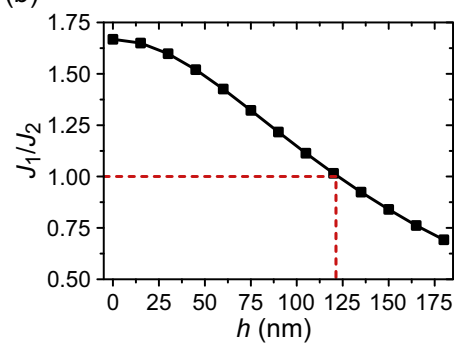

FIG. 1. (a) Three-dimensional representation of a square lattice in which one of the two sublattices (in blue) is shifted vertically by an offset $h$. The nearest-neighbor couplings $J_{1}$ and $J_{2}$ are indicated in red and green, respectively. (b) Using micromagnetic simulations, the $J_{1} / J_{2}$ ratio is calculated as a function of the height offset $h$. For a critical (numerical) value $h_{C}^{\text {num }}$ close to $120 \mathrm{~nm}$, the square ice condition $J_{1} / J_{2}=1$ is obeyed.

microscopy. The magnetic structure factor (MSF), averaged over the different experiments, was computed and intensity profiles in peculiar wave-vector directions of the average MSF were analyzed. Comparing these intensity profiles with those deduced numerically from Monte Carlo simulations, we conclude that long-range magnetostatic interactions, extending beyond nearest neighbors, are not washed out in our lattices and impact the magnetic correlations within or at the vicinity of the ice manifold.

\section{EXPERIMENTAL RESULTS}

The sample consists of square lattices made of 840 Permalloy $500 \times 100 \times 30 \mathrm{~nm}^{3}$ nanomagnets in which one of the two sublattices is shifted vertically [see Fig. 2(a)]. The height offset is set to $h^{\exp }=100 \mathrm{~nm}$, for which the liquid phase was already observed [10]. To shift vertically one of the two sublattices, a two-step electron-beam lithography process was employed: The first step is dedicated to the design of the

nonmagnetic bases, whereas the second one is used for the fabrication of the magnetic arrays. The 30-nm-thick Permalloy layer is capped with a 3-nm-thick aluminum film to limit oxidation.

Because of the nanomagnets' dimensions, the sample is athermal and have thus been demagnetized in a rotating, slowly decaying magnetic field to bring the lattice into a lowenergy magnetic state. The sample is typically demagnetized in three days. After the demagnetization field protocol, the resulting magnetic configuration is imaged using magnetic force microscopy. An image is reported in Fig. 2(b). Analysis of the magnetic contrast at each vertex site allows the determination of the global spin configuration, each nanomagnet being approximated by an Ising variable (i.e., we neglect the magnetization texture within the nanomagnets) [see Fig. 2(c)]. This spin configuration is Fourier transformed to obtain the associated MSF [see Fig. 2(d)].

As reported previously [10], the MSF shares all the features of the low-energy manifold of the square ice model: the background intensity is diffuse but structured and exhibits emergent pinch points for certain wave vectors $[10,26]$. Our measurements were reproduced several times, and a spin liquid state (i.e., a disordered but correlated magnetic configuration $[2,10,31])$ appears systematically in our lattices. The MSF of eight magnetic images were then averaged to improve the statistics [see Fig. 3(a)]. We note that the spin configurations always contain a significant fraction (about $8 \%)$ of magnetic monopoles [see purple and green vertices in Fig. 2(c)], i.e., local spin configurations having an energy higher than the one satisfying the so-called ice (or BernalFowler) rule [44]. The presence of magnetic monopoles reflects the fact that the arrested spin configurations are not in the ground-state manifold.

At first sight the physics we image in our lattices [see Fig. 3(a)] strongly resembles that of the celebrated square ice model [see Fig. 3(b)]. However, careful inspection of the magnetic structure factor reveals features that cannot be accounted for by the square ice model. In particular, additional features (a)

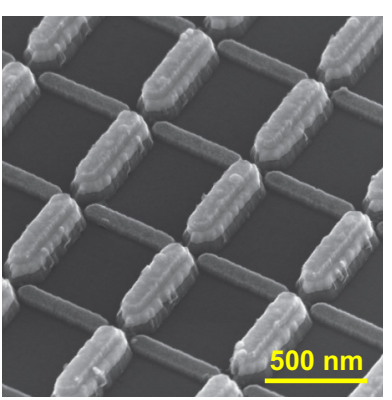

(b)

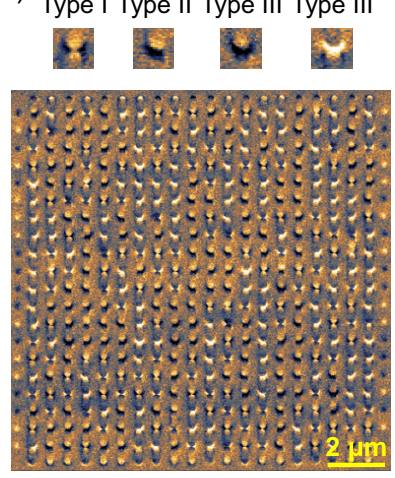

(c)

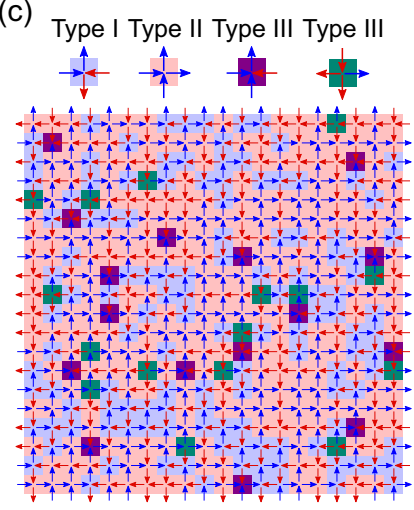

(d)

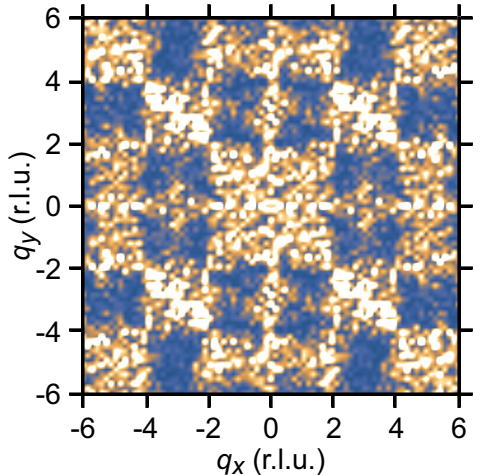

FIG. 2. (a) Electron micrograph of an artificial square ice system in which one sublattice is shifted vertically. (b) Magnetic image of a square lattice with a height offset $h=100 \mathrm{~nm}$. The black and white contrast allows unambiguous determination of the spin configuration. (c) Spin configuration deduced from (b) with a color code highlighting the vertex types. The arrows indicate the direction of the magnetization within each nanomagnet, which are approximated as Ising variables. (d) Magnetic structure factor computed from (c). This magnetic structure factor strongly resembles that of the square ice. 

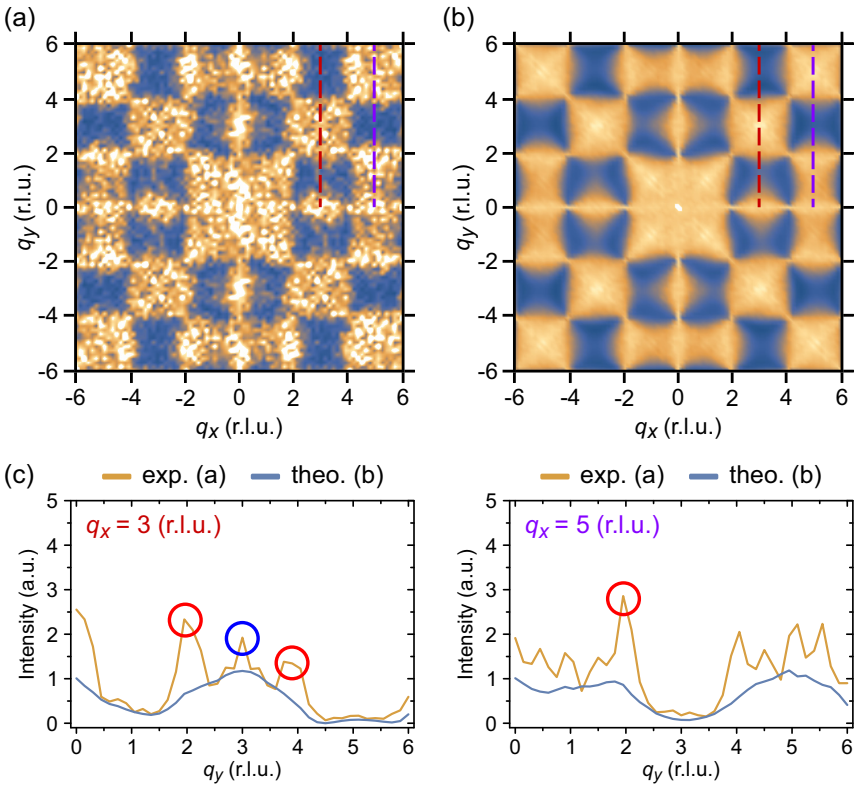

FIG. 3. (a) Experimental magnetic structure factor averaged over eight measurements (two lattices were demagnetized four times). (b) Magnetic structure factor corresponding to the low-energy manifold of the square ice model. The two structure factors cover \pm 6 r.l.u. (reciprocal lattice units). (c) Intensity profiles obtained from (a) (in orange) and (b) (in blue) for the two $q$ directions $q_{x}=3$ and $q_{x}=5$ r.l.u. The scan directions are shown in (a, b) as red ( $q_{x}=3$ r.l.u.) and purple $\left(q_{x}=5\right.$ r.l.u. $)$ dashed lines. The red and blue circles highlight the additional features observed experimentally.

appear along the $q_{x}=3$ and $q_{x}=5$ directions [marked by colored circles in Fig. 3(c)] that are absent in the theoretical MSF. The (short-range) square ice model thus shows limitations in describing the physics observed experimentally. We will show below that these features originate from farther neighbor couplings, i.e., interactions that extend beyond nearest-neighbor nanomagnets.

\section{ORIGIN OF THE ADDITIONAL FEATURES IN THE EXPERIMENTAL MSF}

At this point it is instructive to remember that dipolar interactions are expected to lift the ice degeneracy in a system with a height offset like ours $[28,29]$. However, the nature of the ground state depends on the value of this height offset [28,29]. When the height offset $h$ is smaller than or equal to a critical value $h_{C}$, the ground state is ordered and antiferromagnetic in the sense of the Rys-F model [45,46]. The associated spin configuration and MSF are represented in Figs. 4(a)-4(c). When $h>h_{C}$, the ground state is also ordered and antiferromagnetic but in the sense of the Slater-KDP model [47]: it then consists of an antiferromagnetic alternation of fully polarized lines [see Figs. 4(d)-4(f)].

Interestingly, we find that the magnetic Bragg peaks in these two ground states are located in reciprocal space precisely where additional features appear in the experimental MSF [see Figs. 3(c), 4(c), and 4(f)]. For example, the Rys-F ground state leads to an intense Bragg peak at $\left(q_{x}, q_{y}\right)=$ $(3,3)$. This fits well with the feature marked by a blue circle (a)

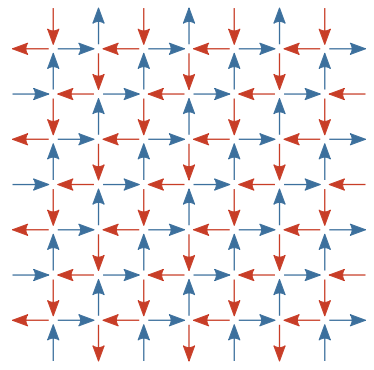

(c)

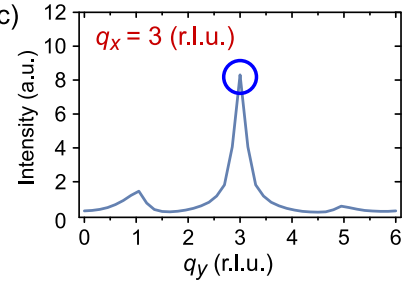

(d)

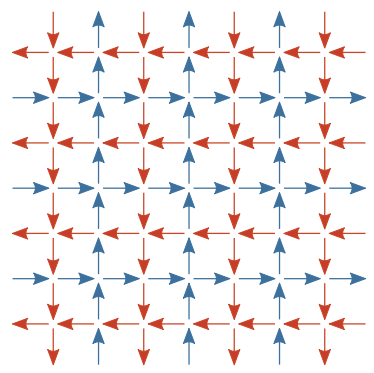

(f)

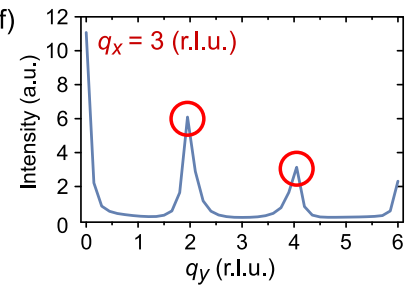

(b)
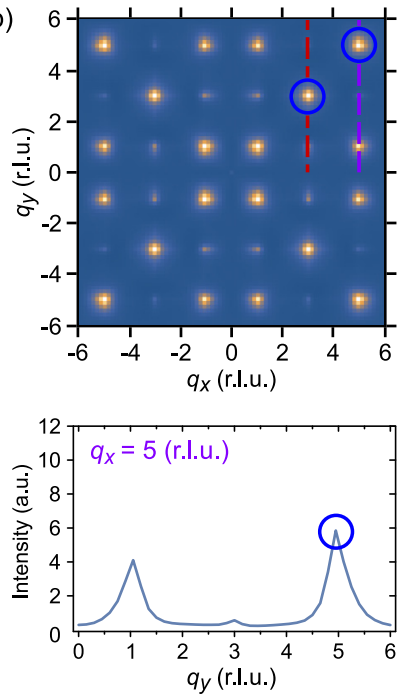

(e)
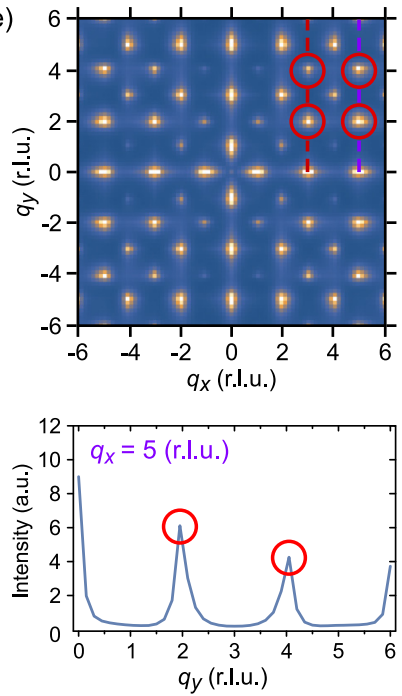

FIG. 4. (a) Real-space and (b) reciprocal-space representation of the ordered ground state of the Rys-F model. (c) Intensity profiles obtained from (b) for the $q_{x}=3$ and 5 r.l.u. directions. (d) Real-space and (e) reciprocal-space representation of the ordered ground state of the Slater-KDP model with an antiferromagnetic coupling between adjacent lines. (f) Intensity profiles obtained from (e) for the $q_{x}=3$ and 5 r.l.u. directions. The two MSF shown here are obtained at finite temperature to make the Bragg peaks broader. The blue and red circles highlight specific wave vectors for which features are observed in the experimental MSF.

in Fig. 3(c). Similarly, the Slater-KDP ground state leads to Bragg peaks at $\left(q_{x}, q_{y}\right)=(3,2),(3,4)$, and $(5,2)$. This also corresponds to the locations marked by a red circle in Fig. 3(c). We note that even the intensity asymmetries between the two pairs of peaks at $(3,2) /(3,4)$ and at $(5,2) /(5,4)$ in Fig. 4(f) are observed in the experimental MSF [see Fig. 3(c)]. The ground-state properties of the dipolar square ice predicted in Refs. [28] and [29] are thus compatible with the features we observe experimentally.

These additional features indicate the presence of extra antiferromagnetic spin-spin correlations on top of the square ice manifold. In other words, the magnetic configurations 


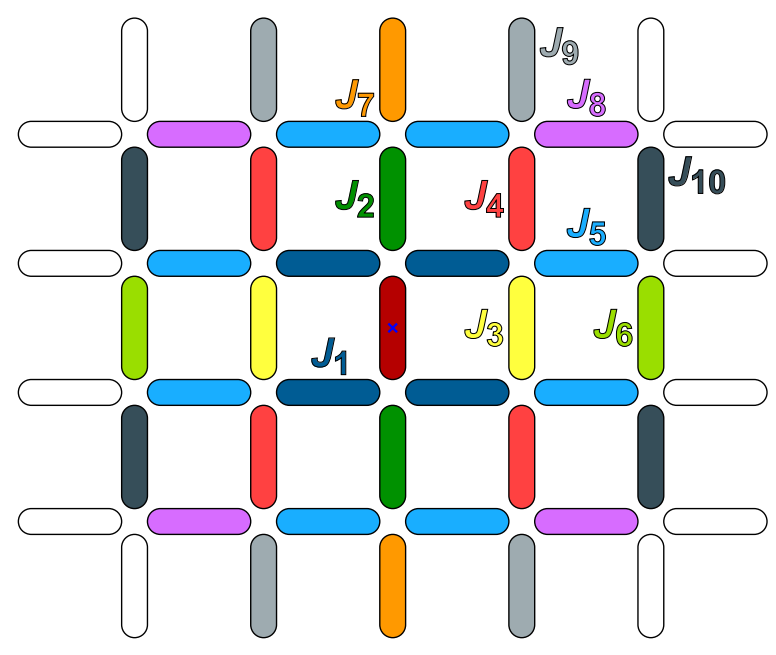

FIG. 5. Two-dimensional map showing the ten coupling strengths considered in this work.

resulting from the field demagnetization of our arrays are not exactly a random arrangement of type-I and type-II vertices. Instead, type-I vertices [appearing in blue in Fig. 2(c)] are slightly more surrounded by other type-I vertices than they should be [i.e., the typical extension of blue clusters made of type-I vertices in Fig. 2(c) is larger than expected in a random distribution]. Type-II vertices [appearing in red in Fig. 2(c)] belonging to adjacent lines in the square lattice are also slightly more antiferromagnetically coupled than expected in the square ice model [see alternations of lines made of red and blue spins in Fig. 2(c)].

\section{BEYOND THE NEAREST-NEIGHBOR DESCRIPTION}

In the previous section we showed that our experimental findings agree qualitatively with the predictions from the dipolar square ice model. To be more quantitative, we now examine the finite temperature properties of an Ising spin Hamiltonian, in which the (magnetostatic) coupling strengths account for the real size of the nanomagnets.

\section{A. Spin Hamiltonian}

To do so, we performed Monte Carlo simulations using the spin Hamiltonian:

$$
H=-\sum_{i, j \mid r_{i j}<\alpha} J_{i j} \sigma_{i} \sigma_{j}
$$

where $\sigma_{i}$ and $\sigma_{j}$ are Ising variables on sites $i$ and $j$, separated by a distance $r_{i j}$, and $\alpha=\sqrt{5} a, a$ being the lattice parameter. Given this $\alpha$ value, we consider coupling strengths extending up to the first ten neighbors (see Fig. 5). The simulations were done for $12 \times 12 \times 2$ lattice sites [48] with periodic boundary conditions using a single spin-flip algorithm. The cooling procedure starts from $T / J_{1}=100$ and ends when the single spin-flip dynamics freezes. $10^{4}$ modified Monte Carlo steps (mmcs) are used for thermalization [49]. Measurements follow the thermalization and are also computed with $10^{4}$ mmcs. The MSF, composed of a matrix of $81 \times 81$ points covering an area of \pm 6 r.l.u. along the $q_{x}$ and $q_{y}$ directions in reciprocal space, is computed as a function of temperature.

\section{B. Coupling strengths}

Although nanomagnets are often considered as Ising pseudospins, they are micromagnetic objects [50-53]. Before computing the thermodynamic properties of the square ice model with farther neighbor interactions, realistic values for the coupling strengths must be estimated. To do so we computed the micromagnetic energy of pairs of nanomagnets having the same dimensions as the ones fabricated experimentally $\left(500 \times 100 \times 30 \mathrm{~nm}^{3}\right.$, with an edge-to-edge distance of $150 \mathrm{~nm}$ between collinear nanomagnets). In a vertically offset lattice, the $J_{1}, J_{5}$, and $J_{8}$ coupling strengths are derived from nanomagnets involving the two sublattices (see Fig. 5). For the other couplings, the two considered nanomagnets belong to the same sublattice. The height offset $h$ then only matters when calculating $J_{1}, J_{5}$, and $J_{8}$.

The micromagnetic energies were computed using the OOMMF code from NIST [54]. The mesh size was set to $1 \times$ $1 \times 15 \mathrm{~nm}^{3}$ to minimize finite difference effects. The small mesh size in the plane of the nanomagnets allows a fairly good description of their round shape extremities. The larger mesh size in the third dimension is chosen to optimize computation time, as it does not substantially affect the estimate of the coupling strengths. We chose the material parameters commonly used for Permalloy: the spontaneous magnetization $M_{s}$ is such that $\mu_{0} M_{s}=1.0053 \mathrm{~T}$, and the exchange stiffness is set to $A=10 \mathrm{pJ} / \mathrm{m}$. Magnetocrystalline anisotropy is neglected, and all simulations are performed at $0 \mathrm{~K}$ (no thermal fluctuations). The coupling strengths $J_{i}(i=1 \ldots 10)$ are derived from the energy difference between a ferromagnetic and an antiferromagnetic configuration of the associated pair of nanomagnets.

First we computed $J_{1}$ as a function of the vertical shift $h$ for values ranging from 0 to $180 \mathrm{~nm}$ by steps of $15 \mathrm{~nm}$. As observed previously [10], $J_{1}$ is larger than $J_{2}$ when $h$ is smaller than a critical value $h_{C}^{\text {num }}$, and smaller otherwise. This dependency of the $J_{1}$ coupling strength with the vertical shift $h$ is reported in Fig. 1(b). A critical value $h_{C}^{\text {num }} \approx 120 \mathrm{~nm}$ is found, consistent with previous estimates [10]. Regarding the other coupling strengths, the results from the micromagnetic simulations are reported in Table I. These coupling strengths are then used in the spin Hamiltonian shown in Eq. (1).

TABLE I. Micromagnetic $J_{i}$ coupling strengths for the first ten neighbors $i$. All values are expressed in arbitrary units (a.u., 1 a.u.=1.05 $\times$ $\left.10^{-18} \mathrm{~J}\right)$. The coupling strengths are calculated at $h^{\exp }=100 \mathrm{~nm}$, i.e., the experimental value of the height offset.

\begin{tabular}{cccccccccccc}
\hline \hline$i$ & 1 & 2 & 3 & 4 & 5 & 6 & 7 & 8 & 9 & 10 \\
\hline$J_{i}$ & 2.220 & 1.933 & -0.333 & 0.045 & 0.104 & -0.052 & 0.132 & 0.073 & 0.058 & -0.018 \\
\hline \hline
\end{tabular}




\section{Results}

Here we report the results from our Monte Carlo simulations and proceed as follows:

(1) We first assume that the square ice condition $J_{1}=J_{2}$ is strictly obeyed.

(2) The thermodynamic properties of the spin Hamiltonian are computed by incorporating the coupling strengths one at a time. A first simulation is performed with $J_{1}$ and $J_{2}$ only, a second one is performed after adding $J_{3}$, a third one after adding $J_{4}$ to the three other values, etc., until all ten micromagnetic $J_{i}$ values reported in Table I are considered.

(3) For all these simulations, intensity profiles along the $q_{x}=3$ and $q_{x}=5$ r.l.u. directions are compared to the experimental ones, similar to what is reported in Fig. 3 for the square ice.

(4) For each simulation, we determine the Monte Carlo temperature that best fits the experimental data.

(5) We then detune the ice condition to check the robustness of our findings.

\section{The $J_{1}=J_{2}$ ice condition}

We first consider the case where $J_{1}=J_{2}=2$ and proceed as described above. The results are reported in Fig. 6 using the same color code as before (the experimental curves are plotted in orange and the simulated ones in blue). The two columns correspond to the $q_{x}=3$ (left) and $q_{x}=5$ (right) r.l.u. directions. The range of coupling strengths included in the spin Hamiltonian is shown on the left, together with the Monte Carlo temperature that best fits the experimental data.

Essentially, these simulations can be grouped in three subsets identified by a colored arrow on the right side of the figure. In the first group (top blue arrow), the main impact of the coupling interactions is to strengthen antiferromagnetic correlations between spins of the same sublattice [marked by a blue circle in Fig. 4(c) for $\left(q_{x}, q_{y}\right)=(3,3)$ and $\left.(5,5)\right]$. Then, farther neighbor couplings (from $J_{5}$ to $J_{8}$, green arrow) affect both the antiferromagnetic correlations between spins of the same sublattice and the antiferromagnetic correlations between neighboring polarized lines [marked by a red circle in Fig. 4(f) for $\left(q_{x}, q_{y}\right)=(3,2),(3,4),(5,2)$, and $\left.(5,4)\right]$. Finally, the two last simulations with $J_{9}$ and $J_{10}$ (bottom blue arrow) show again a preferential selection of antiferromagnetic correlations between spins of the same sublattice.

Overall, the results reported in Fig. 6 show that the incorporation of magnetostatic couplings extending beyond nearest neighbors allow to reproduce qualitatively [see Fig. 6(c)], and even sometimes semiquantitatively [see Figs. 6(e) and 6(f)], the experimental findings. If the other cases fail to capture all the features observed in the experimental MSF, they always capture some of them.

We might wonder why the semiquantitative agreement obtained for coupling strengths extending up to $J_{8}$ [Fig. 6(f)] is lost after incorporating farther neighbor interactions. This is in fact expected [28,29], as we know that a full dipolar treatment of our spin Hamiltonian leads to an ordered antiferromagnetic ground state [illustrated in Figs. 4(a)-4(c)]. Being more quantitative thus requires an additional ingredient that favors antiferromagnetic correlations between neighboring polarized
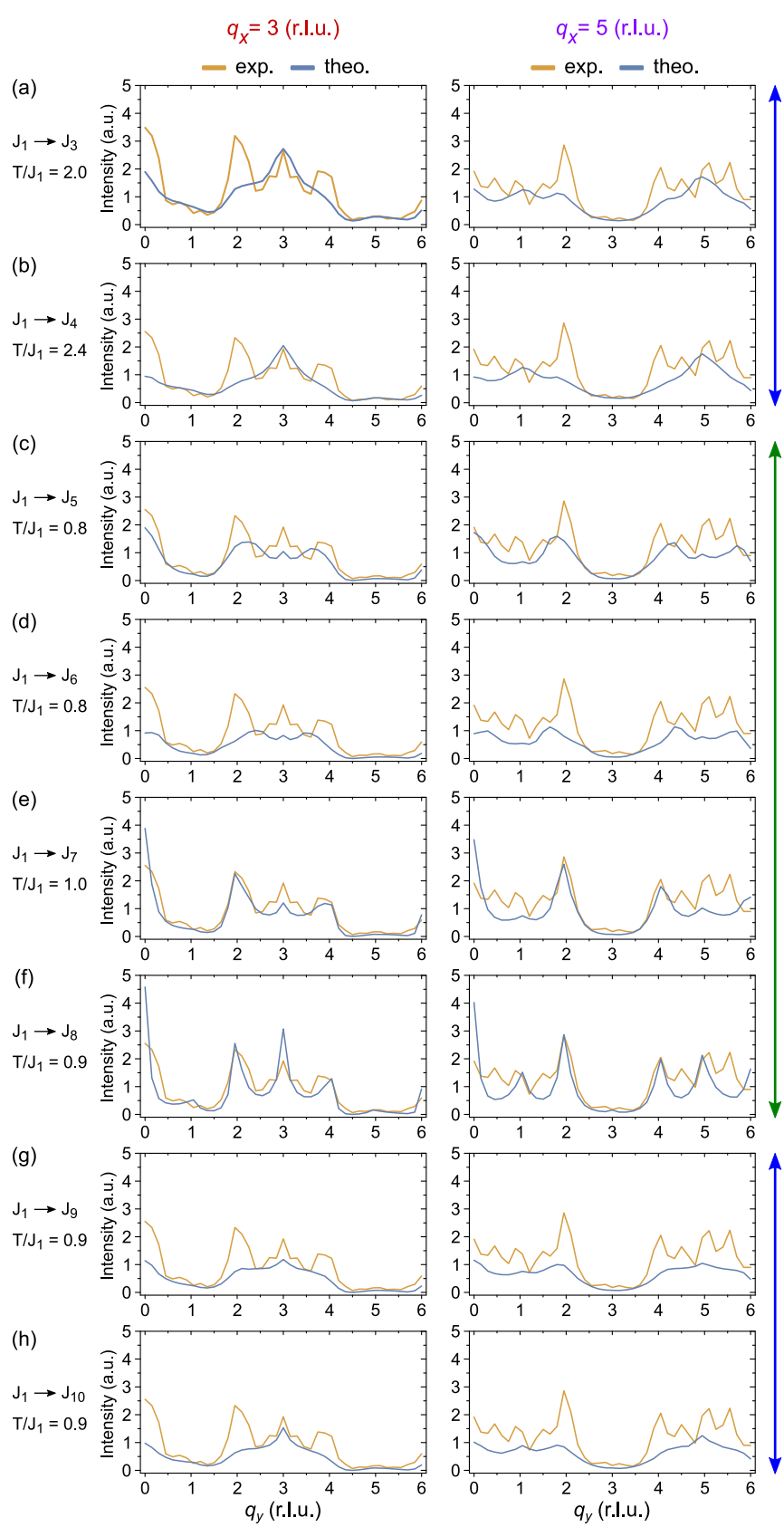

FIG. 6. Intensity profiles in the $q_{x}=3$ and $q_{x}=5$ r.l.u. directions obtained from the magnetic structure factors computed for the spin Hamiltonian with interactions up to the third (a), fourth (b), fifth (c), sixth (d), seventh (e), eighth (f), ninth (g), and tenth (h) coupling strengths. The theoretical scans, shown in blue, are the best fits to the experimental data (orange scans). The Monte Carlo temperature associated to the numerical scans is provided. The blue and green arrows on the right are used to sort the intensity profiles in three different groups (see text for details).

lines. Such magnetic correlations can be induced naturally by slightly detuning the ice condition.

\section{Ice condition detuned: $J_{1}<J_{2}$}

If one can reasonably consider that the ice condition is approached experimentally with a height offset $h=100 \mathrm{~nm}$, it is 

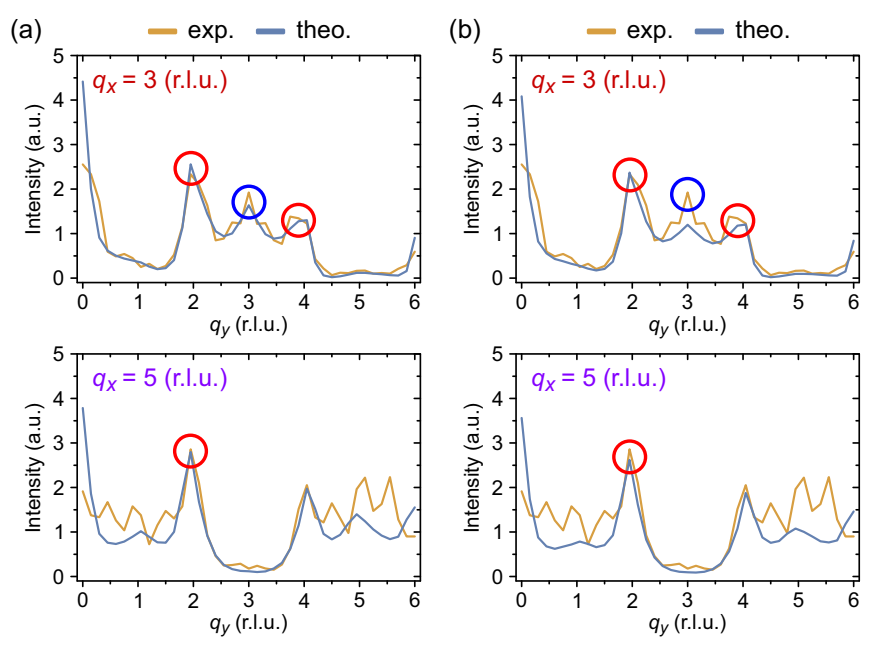

FIG. 7. Intensity profiles along the $q_{x}=3$ and 5 r.l.u. directions for two spin models: (a) $J_{1}=2, J_{2}=2.1, J_{3}=-0.333$, and (b) $J_{1}=$ $2, J_{2}=2.2, J_{3}=-0.333, J_{4}=0.045$. The temperature in the Monte Carlo simulations is $T=1.5 J_{1}$. The blue and red circles highlight the wave vectors for which features are observed in the experimental MSF.

unlikely that is it strictly obeyed. The fact that lithographically patterned arrays are not ideal systems, for example, because of the sample imperfections (roughness, structural or magnetic defects, local misalignment of the nanomagnets, fluctuations in the height offset value, etc.) makes the ice condition in practice hardly achievable. We can then wonder whether the above description is robust when the $J_{1}=J_{2}$ condition is detuned.

The ice condition can be detuned by considering that $J_{1}$ is larger or smaller than $J_{2}$. Obviously, if $J_{1}>J_{2}$, both the detuning condition and the long-range magnetostatic interactions will favor the antiferromagnetic ground state shown in Figs. 4(a)-4(c). This approach can only make the agreement with the experimental MSF less quantitative. However, considering the $J_{1}<J_{2}$ condition will strengthen the antiferromagnetic correlations between neighboring polarized lines, as shown in Figs. 4(d)-4(f). This detuning condition will thus compete with the effect of long-range magnetostatic couplings. Indeed, we find a semiquantitative agreement with the experimental data when choosing $J_{1}=2, J_{2}=2.1$, and $J_{3}=-0.33$ [see Fig. 7(a)]. Note that the agreement is poor if $J_{1}=J_{2}$ [see Fig. 6(a)], whereas it is semiquantitative after detuning the ice condition by about $5 \%$. The same approach works as well with $J_{3}=-0.33$ and $J_{4}=0.045$ after setting $J_{1}=2, J_{2}=2.2$ [see Fig. 7(b)], and works beyond for the very same reasons.

The origin of the additional peaks we observe experimentally is thus further confirmed to be linked to magnetostatic interactions that extend beyond nearest neighbors. We note that the $J_{1}<J_{2}$ detuning condition could be justified in our artificial lattices: (i) if the experimental height offset $h=100 \mathrm{~nm}$ was slightly higher than the numerical critical value $h_{C}^{\text {num }}$, which is, in principle, not the case [see Fig. 1(b)], and

(ii) if the kinetics associated with the spin dynamics when the sample is field demagnetized artificially increase the impact of $J_{2}$, as it is likely (during the field protocol, magnetization reverses via an avalanche process that favors the formation of polarized lines in the lattice, see Ref. [10]).

\section{Ordering vs dynamical freezing}

Finally, the question arises why our system does not order as the Monte Carlo simulations predict [28,29] and why the experimental MSF strongly resembles the one of the square ice, at least at first sight. One reason is the inability of field demagnetization protocols to bring artificial frustrated magnets into a very low-energy state, either because of intrinsic (e.g., dynamical freezing, see $[55,56]$ ) or extrinsic (i.e., quenched disorder) effects. The effective temperature of the spin configurations imaged after our demagnetization procedure thus remains high (see the Monte Carlo temperatures that best fit the experimental data in Fig. 6). Whether artificial lattices are field demagnetized or thermally annealed, exploring the ground-state manifold of an artificial ice magnet is a challenging task [2]. Since the effective temperature of our arrays remains of the order of $J_{1}$, magnetic correlations start to develop and we only observe the emergence of the Bragg peaks characterizing the ground state.

\section{CONCLUSION}

The main result of this work is the experimental evidence of farther neighbor interactions in an artificial square ice. We emphasize again that considering $J_{1}$ and $J_{2}$ only is not sufficient to interpret our results, and considering longer ranged couplings is mandatory, whether the ice condition is obeyed or detuned. As a consequence, the extensive degeneracy of the square ice manifold is expected to be lifted experimentally, provided that we can approach the ground state. This has an important implication for the monopoles' properties. If magnetic monopoles are deconfined quasiparticles, free to diffuse in a pure ice manifold [10,57], they should be bound particles in an ice manifold perturbed by dipolar interactions and should ultimately become confined charged defects in the ground state. In other words, the type-III vertices we observe in the disordered spin configurations we image [see Fig. 2(c)] should behave as weakly bound magnetic monopoles, i.e., the line tension linking a monopole/antimonopole pair is not strictly zero [58-60].

\section{ACKNOWLEDGMENTS}

This work was supported by the Agence Nationale de la Recherche through Project No. ANR-17-CE24-0007-03 "BioIce," and its infrastructure is partially supported by the CAS (Grant No. RVO:68081731).
[1] C. Nisoli, R. Moessner, and P. Schiffer, Rev. Mod. Phys. 85, 1473 (2013).
[2] N. Rougemaille and B. Canals, Eur. Phys. J. B 92, 62 (2019). 
[3] S. H. Skjærvø, C. H. Marrows, R. L. Stamps, and L. J. Heyderman, Nat. Rev. Phys. 2, 13 (2020).

[4] M. J. P. Gingras, Spin ice, in Highly Frustrated Magnetism, edited by C. Lacroix, P. Mendels, and F. Mila (Springer, New York, 2009).

[5] S. T. Bramwell, M. J. P. Gingras and P. C. W. Holdsworth, Spin ice, in Frustrated Spin Systems, edited by H. T. Diep (World Scientific, Singapore, 2013).

[6] I. Gilbert, G.-W. Chern, S. Zhang, L. O’Brien, B. Fore, C. Nisoli, and P. Schiffer, Nat. Phys. 10, 671 (2014).

[7] A. Farhan, C. F. Petersen, S. Dhuey, L. Anghinolfi, Q. H. Qin, M. Saccone, S. Velten, C. Wuth, S. Gliga, P. Mellado, M. J. Alava, A. Scholl, and S. van Dijken, Nat. Commun. 8, 005 (2017).

[8] D. Shi, Z. Budrikis, A. Stein, S. A. Morley, P. D. Olmsted, G. Burnell, and C. H. Marrows, Nat. Phys. 14, 309 (2018).

[9] A. May, M. Hunt, A. V. D. Berg, A. Hejazi, and S. Ladak, Commun. Phys. 2, 13 (2019).

[10] Y. Perrin, B. Canals, and N. Rougemaille, Nature (London) 540, 410 (2016)

[11] Y. Li, G. W. Paterson, G. M. Macauley, F. S. Nascimento, C. Ferguson, S. A. Morley, M. C. Rosamond, E. H. Linfield, D. A. MacLaren, R. Macêdo, C. H. Marrows, S. McVitie, and R. L. Stamps, ACS Nano 13, 2213 (2019).

[12] M. Saccone, A. Scholl, S. Velten, S. Dhuey, K. Hofhuis, C. Wuth, Y.-L. Huang, Z. Chen, R. V. Chopdekar, and A. Farhan, Phys. Rev. B 99, 224403 (2019).

[13] M. Massouras, D. Lacour, M. Hehn, and F. Montaigne, Phys. Rev. B 101, 174421 (2020).

[14] J. Drisko, T. Marsh, and J. Cumings, Nat. Commun. 8, 14009 (2017).

[15] V. Kapaklis, U. B. Arnalds, A. Farhan, R. V. Chopdekar, A. Balan, A. Scholl, L. J. Heyderman, and B. Hjörvarsson, Nat. Nanotechnol. 9, 514 (2014).

[16] I. A. Chioar, B. Canals, D. Lacour, M. Hehn, B. Santos Burgos, T. O. Menteş, A. Locatelli, F. Montaigne, and N. Rougemaille, Phys. Rev. B 90, 220407(R) (2014).

[17] X. Zhang, Y. Lao, J. Sklenar, N. S. Bingham, J. T. Batley, J. D. Watts, C. Nisoli, C. Leighton, and P. Schiffer, APL Mater. 7, 111112 (2019).

[18] D. Louis, D. Lacour, M. Hehn, V. Lomakin, T. Hauet, and F. Montaigne, Nat. Mater. 17, 1076 (2018).

[19] R. Streubel, N. Kent, S. Dhuey, A. Scholl, S. Kevan, and P. Fischer, Nano Lett. 18, 7428 (2018).

[20] N. Leo, S. Holenstein, D. Schildknecht, O. Sendetskyi, H. Luetkens, P. M. Derlet, V. Scagnoli, D. Lançon, J. R. L. Mardegan, T. Prokscha, A. Suter, Z. Salman, S. Lee, and L. J. Heyderman, Nat. Commun. 9, 2850 (2018).

[21] R. F. Wang, C. Nisoli, R. S. Freitas, J. Li, W. McConville, B. J. Cooley, M. S. Lund, N. Samarth, C. Leighton, V. H. Crespi, and P. Schiffer, Nature (London) 439, 303 (2006).

[22] J. P. Morgan, A. Stein, S. Langridge, and C. H. Marrows, Nat. Phys. 7, 75 (2011).

[23] A. Farhan, P. M. Derlet, A. Kleibert, A. Balan, R. V. Chopdekar, M. Wyss, J. Perron, A. Scholl, F. Nolting, and L. J. Heyderman, Phys. Rev. Lett. 111, 057204 (2013).

[24] J. M. Porro, A. Bedoya-Pinto, A. Berger, and P. Vavassori, New J. Phys. 15, 055012 (2013).

[25] E. Östman, H. Stopfel, I.-A. Chioar, U. B. Arnalds, A. Stein, V. Kapaklis, and B. Hjörvarsson, Nat. Phys. 14, 375 (2018).
[26] A. Farhan, M. Saccone, C. F. Petersen, S. Dhuey, R. V. Chopdekar, Y.-L. Huang, N. Kent, Z. Chen, M. J. Alava, T. Lippert, A. Scholl, and S. van Dijken, Sci. Adv. 5, eaav6380 (2019).

[27] E. H. Lieb, Phys. Rev. 162, 162 (1967).

[28] G. Möller and R. Moessner, Phys. Rev. Lett. 96, 237202 (2006).

[29] L. A. S. Mól, W. A. Moura-Melo, and A. R. Pereira, Phys. Rev. B 82, 054434 (2010).

[30] G.-W. Chern, M. J. Morrison, and C. Nisoli, Phys. Rev. Lett. 111, 177201 (2013).

[31] Y. Perrin, B. Canals, and N. Rougemaille, Phys. Rev. B 99, 224434 (2019).

[32] Y. Qi, T. Brintlinger, and J. Cumings, Phys. Rev. B 77, 094418 (2008).

[33] G. Möller and R. Moessner, Phys. Rev. B 80, 140409(R) (2009).

[34] N. Rougemaille, F. Montaigne, B. Canals, A. Duluard, D. Lacour, M. Hehn, R. Belkhou, O. Fruchart, S. El Moussaoui, A. Bendounan, and F. Maccherozzi, Phys. Rev. Lett. 106, 057209 (2011).

[35] S. Zhang, I. Gilbert, C. Nisoli, G.-W. Chern, M. J. Erickson, L. OBrien, C. Leighton, P. E. Lammert, V. H. Crespi, and P. Schiffer, Nature (London) 500, 553 (2013).

[36] M. E. Brooks-Bartlett, S. T. Banks, L. D. C. Jaubert, A. Harman-Clarke, and P. C. W. Holdsworth, Phys. Rev. X 4, 011007 (2014).

[37] F. Montaigne, D. Lacour, I. A. Chioar, N. Rougemaille, D. Louis, S. Mc Murtry, H. Riahi, B. Santos Burgos, T. O. Mentes, A. Locatelli, B. Canals, and M. Hehn, Sci. Rep. 4, 5702 (2014).

[38] B. Canals, I. A. Chioar, V. D. Nguyen, M. Hehn, D. Lacour, F. Montaigne, A. Locatelli, T. O. Menteş, B. Santos Burgos, and N. Rougemaille, Nat. Commun. 7, 11446 (2016).

[39] I. A. Chioar, N. Rougemaille, A. Grimm, O. Fruchart, E. Wagner, M. Hehn, D. Lacour, F. Montaigne, and B. Canals, Phys. Rev. B 90, 064411 (2014).

[40] I. A. Chioar, N. Rougemaille, and B. Canals, Phys. Rev. B 93, 214410 (2016).

[41] J. Hamp, R. Moessner, and C. Castelnovo, Phys. Rev. B 98, 144439 (2018).

[42] A. S. Wills, R. Ballou, and C. Lacroix, Phys. Rev. B 66, 144407 (2002).

[43] S. Zhang, J. Li, I. Gilbert, J. Bartell, M. J. Erickson, Y. Pan, P. E. Lammert, C. Nisoli, K. K. Kohli, R. Misra, V. H. Crespi, N. Samarth, C. Leighton, and P. Schiffer, Phys. Rev. Lett. 109, 087201 (2012).

[44] J. D. Bernal and R. H. Fowler, J. Chem. Phys. 1, 515 (1933).

[45] F. Rys, Helv. Phys. Acta 36, 537 (1963).

[46] E. H. Lieb, Phys. Rev. Lett. 18, 1046 (1967).

[47] E. H. Lieb, Phys. Rev. Lett. 19, 108 (1967).

[48] We checked that our results do not depend on the system size. In specific cases, simulations were run for $12 \times 12 \times 2,20 \times$ $20 \times 2$ and $30 \times 30 \times 2$ lattice sites. No substantial difference was observed.

[49] One modified Monte Carlo step corresponds to a sequence of spin-flip attempts such that, on average, each spin is flipped once. In practice, this corresponds to a number of trials equal to $N$ (number of sites) $\times$ the inverse of the acceptance rate (average value of the spin-flip acceptance). At the lowest temperatures, $10^{4} \mathrm{mmcs}$ corresponds typically to $10^{7}$ spin-flip trials. This allows to achieve statistical independence. 
[50] N. Rougemaille, F. Montaigne, B. Canals, M. Hehn, H. Riahi, D. Lacour, and J.-C. Toussaint, New J. Phys. 15, 035026 (2013).

[51] S. Gliga, A. Kákay, L. J. Heyderman, R. Hertel, and O. G. Heinonen, Phys. Rev. B 92, 060413(R) (2015).

[52] V.-D. Nguyen, Y. Perrin, S. Le Denmat, B. Canals, and N. Rougemaille, Phys. Rev. B 96, 014402 (2017).

[53] G. W. Paterson, G. M. Macauley, Y. Li, R. Macêdo, C. Ferguson, S. A. Morley, M. C. Rosamond, E. H. Linfield, C. H. Marrows, R. L. Stamps, and S. McVitie, Phys. Rev. B 100, 174410 (2019).

[54] M. Donahue and D. Porter, Interagency Report NISTIR 6376, National Institute of Standards and Technology, Gaithersburg, MD, 1999.
[55] V. Schánilec, B. Canals, V. Uhlîr, L. Flajšman, J. Sadílek, T. Šikola, and N. Rougemaille, Phys. Rev. Lett. 125, 057203 (2020).

[56] R. G. Melko and M. J. P. Gingras, J. Phys: Condens. Matter 16, R1277 (2004).

[57] C. Castelnovo, R. Moessner, and S. L. Sondhi, Nature (London) 451, 42 (2008).

[58] L. A. Mól, R. L. Silva, R. C. Silva, A. R. Pereira, W. A. MouraMelo, and B. V. Costa, J. Appl. Phys. 106, 063913 (2009).

[59] R. C. Silva, F. S. Nascimento, L. A. S. Mól, W. A. Moura-Melo, and A. R. Pereira, New J. Phys. 14, 015008 (2012).

[60] F. S. Nascimento, L. A. S. Mól, W. A. Moura-Melo, and A. R. Pereira, New J. Phys. 14, 115019 (2012). 